\title{
Energetic Competition in the Complexation Affinity of Paracetamol with Water and Oxalic Acid
}

\author{
Amanda Studinger, Loredana Valenzano-Slough* \\ Michigan Technological University, Department of Chemistry, Houghton, MI 49931, USA
}

Keywords:

DFT, intermolecular interactions, long-range forces, weak forces, hydrogen bond, molecular complexation, molecular affinity, relative stability, active pharmaceutical ingredients, paracetamol, nucleation, crystal growth.

*Corresponding Author: lvalenza@mtu.edu 


\section{Abstract}

The intermolecular interactions of active pharmaceutical ingredients (APIs) are the driving forces of API molecular activity in solid crystal structure and stability, in vivo receptor binding, and in environmental transport and persistence. In API manufacturing, complexation of the API with another molecule (cocrystal conformer) to create stable crystalline structures can be a useful tool, but the possibility of more energetically stable complexes forming instead of the target conformation can lead to undesired changes to the properties of the final product. Natural complexation of the API to cocrystal conformers in the environment can cause undesirable accumulation of the API. Using Density Functional Theory (DFT), the paracetamol and oxalic acid (PCA-OXA) cocrystal was studied to determine how hydrogen bonding dictates complex formation. Five different functionals were employed: B3LYP-D3, B3LYP-D3-BJ, M06-2X, M062X-D3, and $\omega$ B97-XD in combination with the aug-cc-pVDZ Dunning basis set. The hydrogen bonding sites of PCA-OXA were investigated to determine strength and overall contribution/competition of H-bonding formation during the initial stages of the nucleation process by relaxing the geometries and evaluating structural and energetic changes. Complexation and configuration energies were calculated for 1:1 PCA-OXA, PCA-Water, and OXA-Water complexes. Calculations were performed both in vacuum and in water environment (explicit and implicit solvation). Results showed significant shortening of the hydrogen bonds within the PCAOXA complex with large changes in energy during complexation of oxalic acid with paracetamol. An overall analysis of all of the results collected for the PCA-OXA complexes indicates that sites 2 and 4 play the largest roles in the initial steps of the nucleation process with position 2 showing the most stable intermolecular interactions. It is expected that in an aqueous environment, there would be little to no energetic competition for PCA to form hydrogen bonds with other molecules. 


\section{Introduction}

Intermolecular interactions such as hydrogen bonds and van der Waals forces are the driving forces for many practical applications of active pharmaceutical ingredients (APIs). In the design and manufacturing process, it is beneficial to produce APIs as cocrystals with another molecule (cocrystal conformers) to create stable crystalline structures that have the desired physicochemical properties. ${ }^{1}$ API cocrystals are also designed to maximize the pharmacokinetic properties of the final product such as absorption, distribution, metabolism, and excretion. In this regard, hydrogen bond nets and interlayered van der Waals forces play a major role in how the API crystals are formed during manufacturing, and how easily they are dried, pelletized or encapsuled, transported and stored. In addition to influencing the pharmacokinetic properties in vivo, the final crystal structure's unique intermolecular interactions and how they are influenced by their surrounding environment can influence the pharmacodynamics of a drug regarding how it binds to targeted receptors. In addition, after the drug is used or metabolized, or when excess drugs enter waste streams, the same intermolecular interactions act again in a wide array of chemical environments in the water and soil with these processes dictating how the APIs are transported and/or stored in the environment. ${ }^{2}$

During the manufacturing process of API solids, the crystals are usually formed when API crystals crash out of the super saturated solution and are subsequently dried and packaged. While this method is commonly used in large scale manufacturing processes, it presents unresolved challenges related to the difficulty in controlling the formation of the actual final crystal structure. In fact, the possibility of more energetically stable complexes forming instead of the target conformation can lead to undesired changes to the physicochemical and/or physiological 
properties of the final product. In addition, more energetically favorable cocrystal conformers can also influence transport and storage of APIs in the environment.

With these premises, acknowledging the critical importance that intermolecular interactions play in the formation, usage, and disposal of APIs, it warrants further efforts to investigate the intimate properties of these interactions, their driving forces, and how they can be compared across several molecular species. Such a small-scale quantitative investigation can be achieved only with the aid of electronic structure methods. The approach chosen for this work relies on density functional theory (DFT) to determine complexation energies, heats of complexation, and several other physicochemical descriptors able to shed light on the complexation affinity of paracetamol with water and oxalic acid. This method will allow for comparing the behavior of the molecular interactions in different chemical environments to get original quantitative insights into the factors influencing these interactions. Such an understanding can allow for more effective drug designs, controlled drug synthesis and crystallization, and more accurate environmental risk assessments.

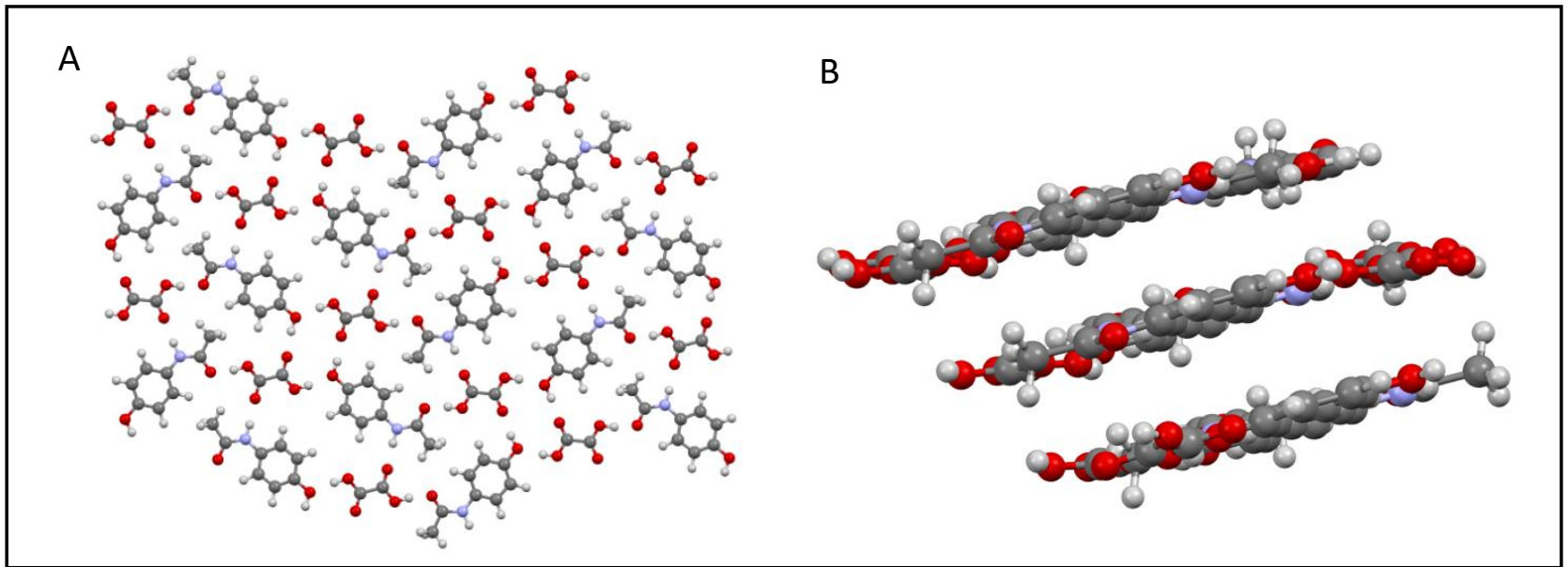

Figure 1. Crystal structure of the PCA-OXA complex with (A) coplanar layers held together by hydrogen bonding and (B) $\pi-\pi$ interlayer stacking. 
The goals of this work include an evaluation of heats of complexation of paracetamol and oxalic acid (PCA-OXA), paracetamol-water (PCA-Water), and oxalic acid-water (OXA-Water) molecular complexes, and a detailed assessment of the hydrogen bonding sites of PCA to determine the forces driving the complexation mechanisms and their effects on the molecules' mutual geometrical arrangement. It is expected that such arrangement will drive the very initial stages of the crystalline nucleation process which, eventually, will drive the growth mechanism of the crystal itself. PCA-OXA appears in a 1:1 stoichiometric ratio. Figure 1 shows the unit cell of the PCA-OXA complex as obtained from the crystal infographic file (CIF). ${ }^{3}$ Given their almost perfect planar nature, oxalic acid and paracetamol molecules form a coplanar hydrogen bond net within the crystal structure. In addition, paracetamol and oxalic acid provide six unique hydrogen bonding sites that allow for an in-depth comparison of how different factors influence the strength and stability of hydrogen bonding.

Results obtained using explicit and implicit solvation are compared. Overall, this work aspires to pose a quantitative basis for the future investigation of the nucleation process of the PCA-OXA cocrystal and, by extension, of other APIs. It is expected that this work can contribute to the understanding of the energetic competition present in any aqueous environment, from the initial manufacturing processes, to applications in biological systems, and finally to distribution in the environment.

\section{Theoretical and Computational Details}

The Gaussian16 program ${ }^{4}$ was used and several Density Functional Theory (DFT) approximations $^{5}$ were chosen to determine relaxed geometries and electronic energies of all molecular complexes reported. Five different functionals were employed: B3LYP-D3 ${ }^{6-8}$, B3LYP- 
D3-BJ, M06-2X ${ }^{9}, \mathrm{M} 06-2 \mathrm{X}-\mathrm{D} 3$, and $\omega \mathrm{B} 97-\mathrm{XD}^{10}$, where $\mathrm{D}^{11}$ and $\mathrm{BJ}^{12}$ refer to a posteriori corrections able to describe long range dispersion forces. The Dunning basis set (aug-cc-pVDZ ${ }^{13}$ ) was used for all calculations. Calculations were performed in vacuum and in a water solvated environment $\left(\mathrm{CPCM}^{14}\right)$. Optimization calculations were performed at $0 \mathrm{~K}$ and harmonic frequency calculations at 298.15 K (and $1 \mathrm{~atm}$ ). Basis set superposition error (BSSE) was determined using the counterpoise correction method. ${ }^{15}, 16$ Default parameters for numerical integration grid accuracy, convergence criteria, and force constants were used with the exception of the PCAWater complex for position 1 in a solvated environment where parameters were adjusted using a superfine grid and adjusted force constants in order to remove the presence of imaginary frequencies.

For comparison purposes, configuration properties $(P)$ refer to values for the physicochemical properties reported in this work. The difference in $P$ is obtained by subtracting values for the complexes as extracted from the crystalline structure from results obtained from the relaxed data. For example, configuration changes in hydrogen bond lengths $(R)$, angles $(A)$, and energies $(E)$ were determined as:

$$
\Delta P=P_{\text {relaxed }}-P_{\text {crystalline }}
$$

Complexation (electronic) energies were obtained by applying Hess' law

$$
\Delta E_{e(\text { complexation })}=E_{e(\text { complex })}-E_{e(\text { sum of fragments })}
$$

and results were further corrected for the BSSE

$$
\Delta E_{e(\text { complexation })}^{B S S}=E_{e(\text { complex })}-E_{e(\text { sum of fragments })}+B S S E
$$

Frequency calculations were performed to obtain the necessary thermochemical data allowing for the determination of heats of complexation (Equation 4). Calculations were performed for all the molecular complexes discussed in this work. Note that the solvated PCA-Water complex 
at position 1 resulted in imaginary frequencies. This numerical instability was cured by tightening the default parameters for grid size, force constants, and convergence criteria. It was determined that the use of the combined keywords $O P T=$ calcall and Integral(grid=superfine) resulted in real frequencies, but at a higher computational cost ( $4 \mathrm{X}$ the cost of a calculation at default parameters). When comparing results with all parameter tests, energy values did not show any statistically significant differences. It was determined that for the purposes of this work, the presence of imaginary frequencies does not influence the energy values of interest and do not necessarily need to be corrected (Table S-1).

Heats (i.e., enthalpies) of complexation were determined by further adding the vibrational zero-point energy contribution and the thermal correction determined as per ideal gas approximation

$$
\Delta H=\Delta E_{e(\text { complexation })}^{B S S E}+\Delta E_{Z P E}+\Delta E_{T}
$$

The unique hydrogen bonding sites that were evaluated for this project were labeled as shown in Figure 2. The PCA-OXA crystal structure data were obtained from the Cambridge Crystallographic Data Center (CCDC) database ${ }^{3}$ (crystal infographic file (CIF) \#720368 ${ }^{17}$ ). From the CIF, the components of interest (PCA, OXA, and PCA-OXA complexes) were isolated and exported from Moldraw ${ }^{18}$ to Guassian $16^{4}$. The Avogadro graphical interface ${ }^{19}$ was then used to visualize the completed calculations and final geometries. Various combinations of PCA-OXA, PCA-Water, and OXA-Water monomers were analyzed. The monomers analyzed consisted of dispecies (1:1) complexes. 


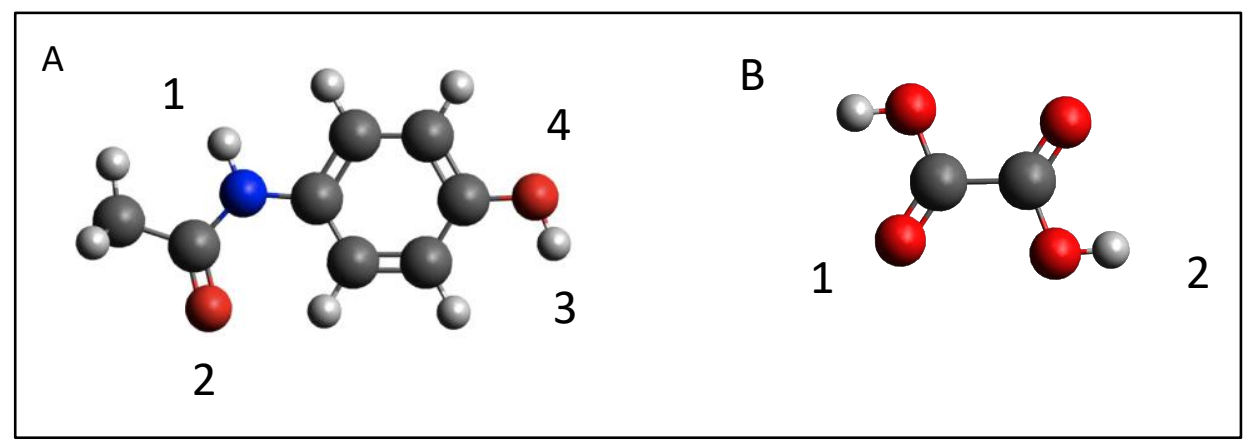

Figure 2. Hydrogen bonding sites labeled as: (A) Positions 1-4 on paracetamol (PCA), (B) Positions 1,2 on oxalic acid (OXA) molecules.

\section{Results and Discussion}

One-to-one hydrogen bonded di-species complexes of paracetamol, oxalic acid, and water were investigated. Initially, single point energy calculations were performed on the four PCAOXA complexes as extracted from the experimentally refined PCA-OXA crystalline structure which provided a reference for the geometrical and energetic complexation of the individual molecular components as they assemble in the final crystalline structure. Subsequently, geometry relaxation calculations were performed on the same four PCA-OXA complexes (both in vacuum and in aqueous implicit solvation) allowing for a quantitative appreciation of the influence provided by the crystalline chemical environment on the complexes themselves. This approach allowed for a detailed analysis of geometrical changes in the individual molecules and complexes (hydrogen bond strengths), and of electronic complexation energies which were further corrected with respect to the basis set superposition error (BSSE). In addition, frequency calculations on the complexes' equilibrium geometries were performed to obtain heats of complexation arising from the inclusion of the thermal correction $(298.15 \mathrm{~K})$ and zero-point vibrational energies (ZPVE). In a similar fashion, PCA-Water and OXA-Water complexes were also studied. Each scenario was 
repeated for each functional using the same basis set (aug-cc-pVDZ). All values reported are average values among the five functionals employed unless explicitly stated otherwise.

\subsection{Geometry}

The crystalline structures of each PCA-OXA complex were relaxed using a self-consistent field (SCF) procedure. Differences in intra- and intermolecular bond lengths and angles between the crystallized and relaxed geometries were evaluated for conformational changes that occurred during complexation. A comparison of the relaxed and crystallized geometries of oxalic acid and paracetamol showed that oxalic acid underwent an appreciable conformational change, the most notable involving decreased bond lengths for the carbon-oxygen single bonds (0.07-0.05 $\AA$, Table S-2 and Table S-3). Changes in bond lengths for PCA were minimal (0.0001 to $0.02 \AA)$ with the most notable changes seen in vacuum at hydrogen bonding position 2 and both vacuum and aqueous environments at position 3 where the bonds were longer in the crystallized conformation by $0.02 \AA$ (Table S-2 and Table S-3). Overall evaluation of PCA shows that the shortened bonds of the $\mathrm{C}=\mathrm{O}$ (position 2) and $\mathrm{O}-\mathrm{H}$ (positions 3 and 4) sites in the relaxed geometry are the most likely contributors to the lower energy configuration of the relaxed paracetamol as compared to the PCA-OXA complex. Changes in angles were most notable at positions 3 and $4\left(-2.5^{\circ}\right.$ and $4.5^{\circ}$ respectively) which can be attributed to the additional space and freedom of movement allowed outside of the crystal structure. 


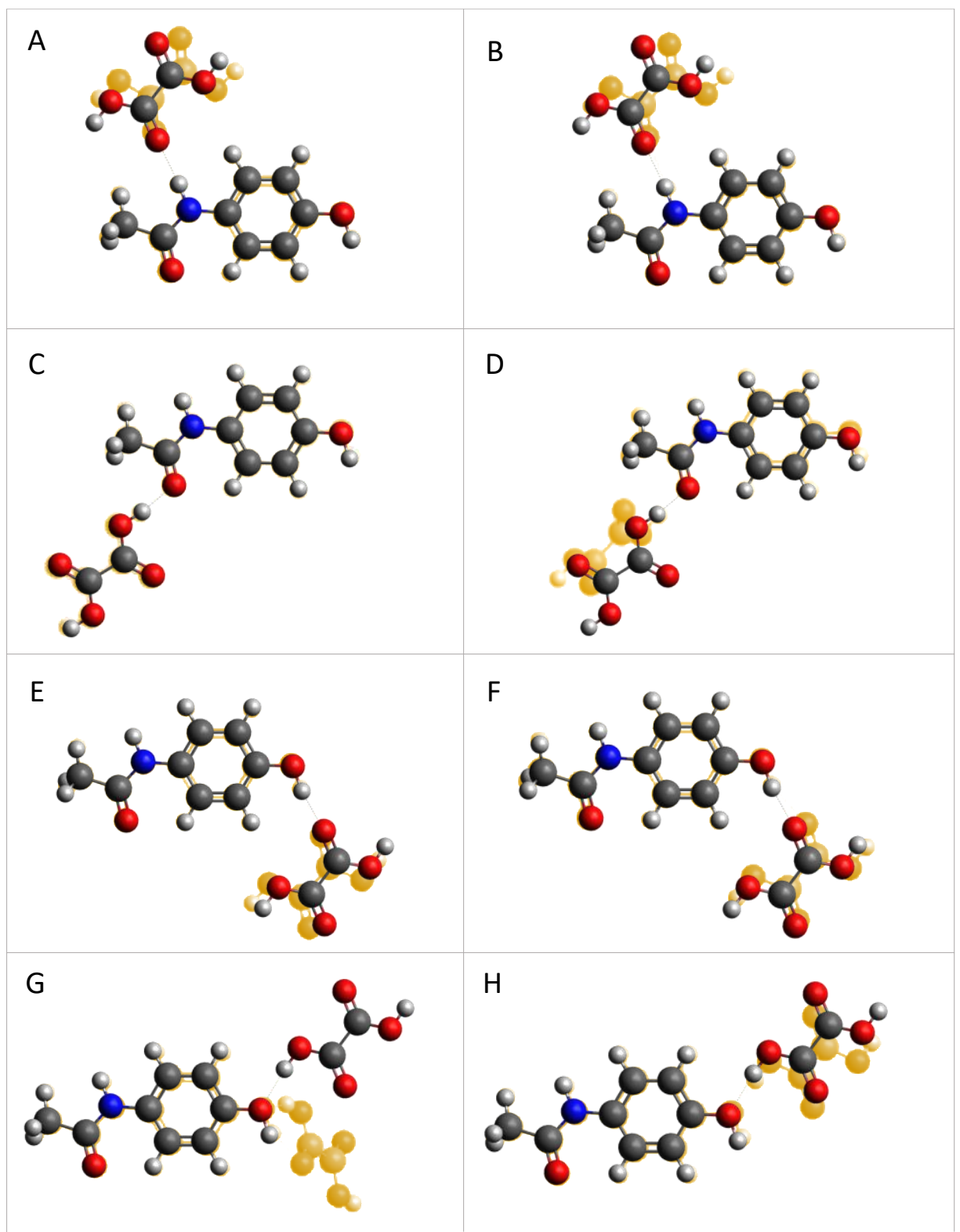

Figure 3. Conformational changes of the PCA-OXA complexes in vacuum and an aqueous environment for (A, B) Position 1, (C, D) Position 2, (E, F) Position 3, and (G, H) Position 4 using B3LYP-D3. The shaded yellow background shows the relaxed geometries.

Figure 3 shows the changes in geometry due to the rotation of the oxalic acid in relation to PCA using the B3LYP-D3 functional. Molecular mutual arrangements among the different functionals were comparable with a few noted exceptions. In vacuum, relaxed geometries for PCAOXA at position 2 were nearly identical with a change in H-bond length of $0.0446 \AA$ with a standard deviation of $0.0106 \AA$ A. But in an aqueous environment, using B3LYP-D3, B3LYP-D3- 
$\mathrm{BJ}$, and $\omega \mathrm{B} 97-\mathrm{XD}$ resulted in a $66.5^{\circ}$ out of plane rotation of oxalic acid with respect to PCA (Figure 3-D). The most varied results occurred at position 4 of PCA-Water and PCA-OXA complexes, both in vacuum and in an aqueous environment (Figure 3-G, Figure S-1, and Figure S-2 ). Here, water and oxalic acid was observed to rotate towards or away from PCA, in or out of plane, and form one or two hydrogen bonds with the aromatic hydroxyl group. While results for positions 1 and 3 were consistent in both vacuum and aqueous environments (Figure 3-A,B,E,F), they varied considerably at positions 2 and 4 . In vacuum, the oxalic acid in position 2 remained in its coplanar configuration (Figure 3-C) but rotated out of plane at position 4 (Figure 3-G). In an aqueous environment the opposite was true; results showed an out of plane rotation at position 2 (Figure 3-D) and a coplanar configuration at position 4 (Figure 3-H). In vacuum, the oxalic acid at position 4 formed two hydrogen bonds with the hydroxyl group of PCA during the optimization process (Figure 3-G). This can be explained by observing that in an aqueous environment, it is possible there is enough of an energetic barrier from the solvent to prevent the formation of the additional hydrogen bond.

The H-bond lengths between PCA and OXA were evaluated for the initial and relaxed geometries of each complex. The largest average relaxed bond lengths at positions 1 and 3 in vacuum ( 0.30 and $0.21 \AA$, respectively) could be an indication of steric strain between the oxalic acid and the PCA aromatic ring as shown in Figure 4. Overall, bond lengths were longer in the relaxed geometries of each complex in vacuum than in an aqueous environment. This can be related to an induced polarity of PCA and oxalic acid from the solvent, which would strengthen the hydrogen bonds and result in shorter bond lengths. These observations agree with the results discussed earlier for oxalic acid, where the overall PCA-OXA in the crystal structure seems to be compressed. This might be an indicator that the attractive forces between the paracetamol 
molecules belonging to different crystalline layers ( $\pi-\pi$ stacking, long range dispersion forces) and possibly that of hydrogen netting between the varying H-bonds drive the overall nucleation of the complex, and in turn, compresses the oxalic acids and the hydrogen bonds.

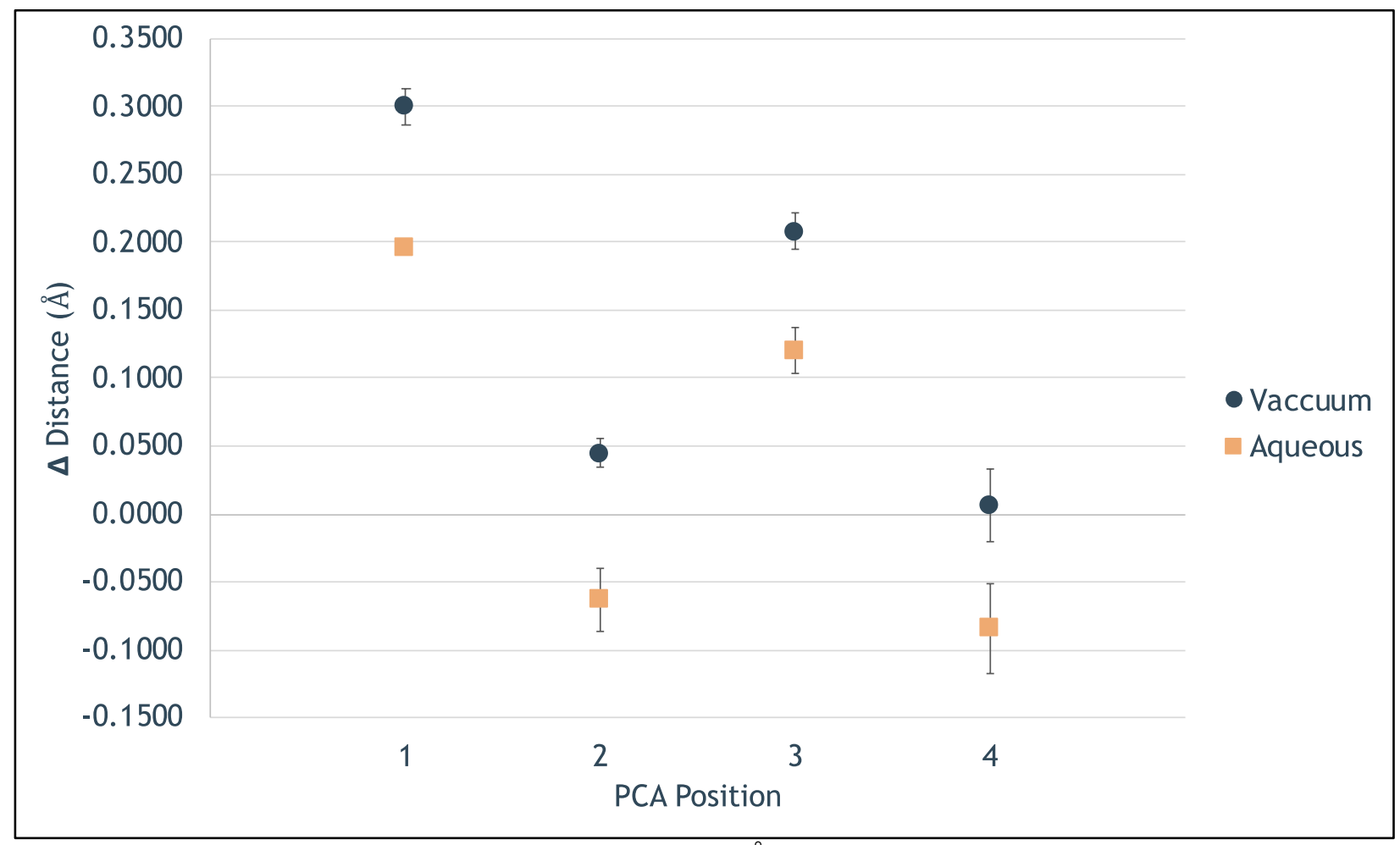

Figure 4. Averaged changes in hydrogen bond lengths $(\AA)$ of the PCA-OXA complexes in vacuum and an aqueous environment.

Changes in the hydrogen bond angles were also evaluated to determine possible trends with respect to geometry stabilization and/or strain within the PCA-OXA complex as shown in Figure 5. In vacuum, the most notable change in angle occurred at position $3\left(13.7^{\circ}\right)$ and to a lesser extent at position $1\left(8.4^{\circ}\right)$. This, again, indicates the presence of steric hindrance with the aromatic ring of PCA. In an aqueous environment, results at positions 3 and 4 showed the most dramatic changes among the several functionals employed (std $9.3^{\circ}$ and $10.6^{\circ}$, respectively). An interesting observation is that the H-bond angles for the M06-2X and M06-2X-D3 were in good agreement with each other (within $0.2^{\circ}$ ) at all positions. Because the M06-2X functional was designed with 
long range dispersion forces in mind, it is not surprising that the additional D3 correction does not have a large effect on the results.

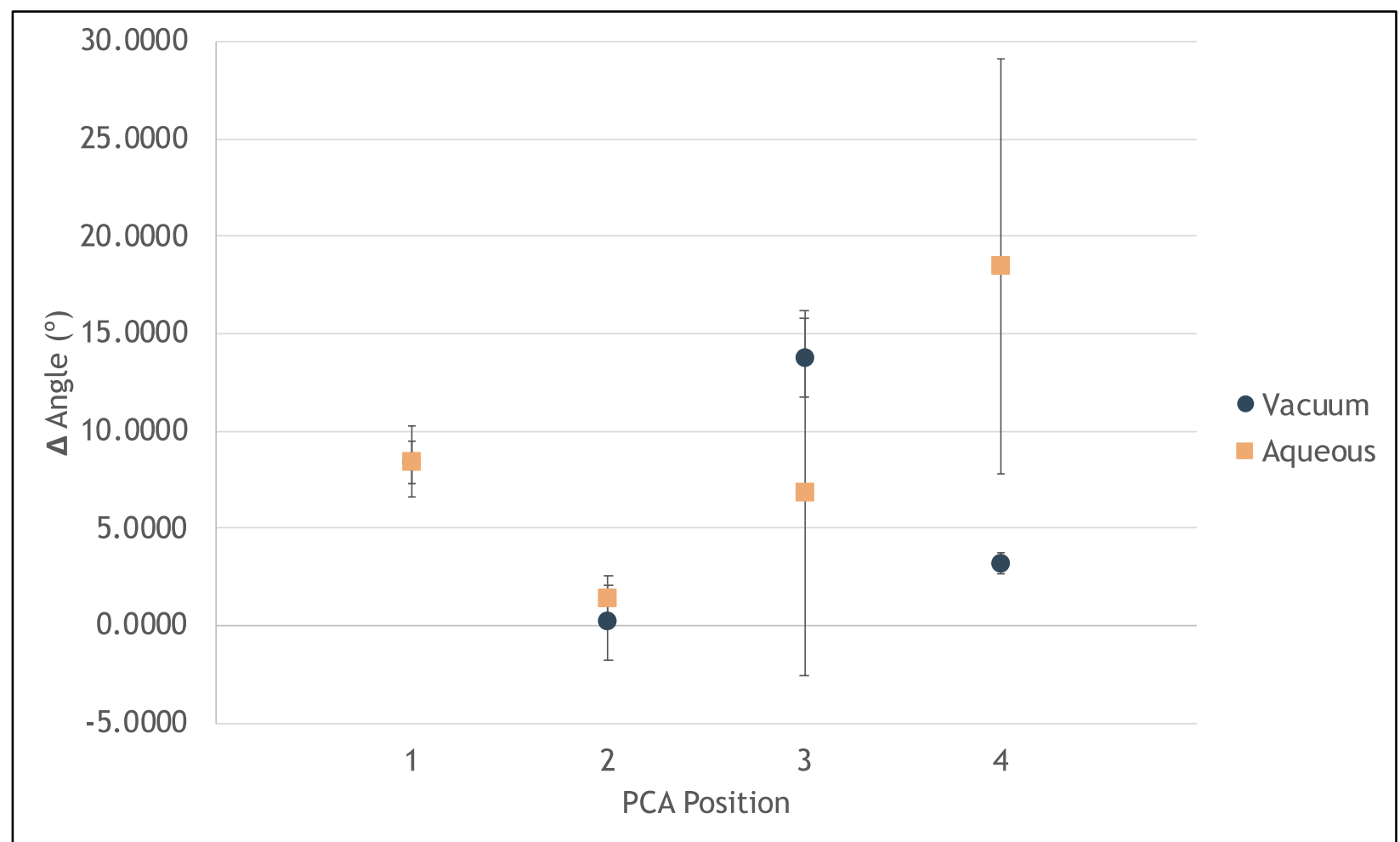

Figure 5. Averaged changes in hydrogen bond angles $\left(^{\circ}\right)$ of the PCA-OXA complexes in vacuum and an aqueous environment.

An overall analysis of all of the results collected for the PCA-OXA complexes, indicates that sites 2 and 4 could play the largest roles in the initial steps of the nucleation process. Considering the compressed geometry of the complex, it is also very likely that other forces such as thermochemistry, $\pi-\pi$ interlayer stacking, heats of complexation, and competition from other possible hydrogen bond formation with PCA, OXA, or impurities play a significant role in driving the nucleation process.

Hydrogen bond lengths were compared for the relaxed geometries of the OXA-Water, PCA-Water, and PCA-OXA complexes in vacuum and in an aqueous environment. In an aqueous environment, the hydrogen bond lengths for every complex were shorter than in vacuum $(0.10 \AA)$ 
as shown in Figure 6. This suggests that the surrounding solvent increases the polarity of the molecules which results in stronger bonds that are shorter in length.

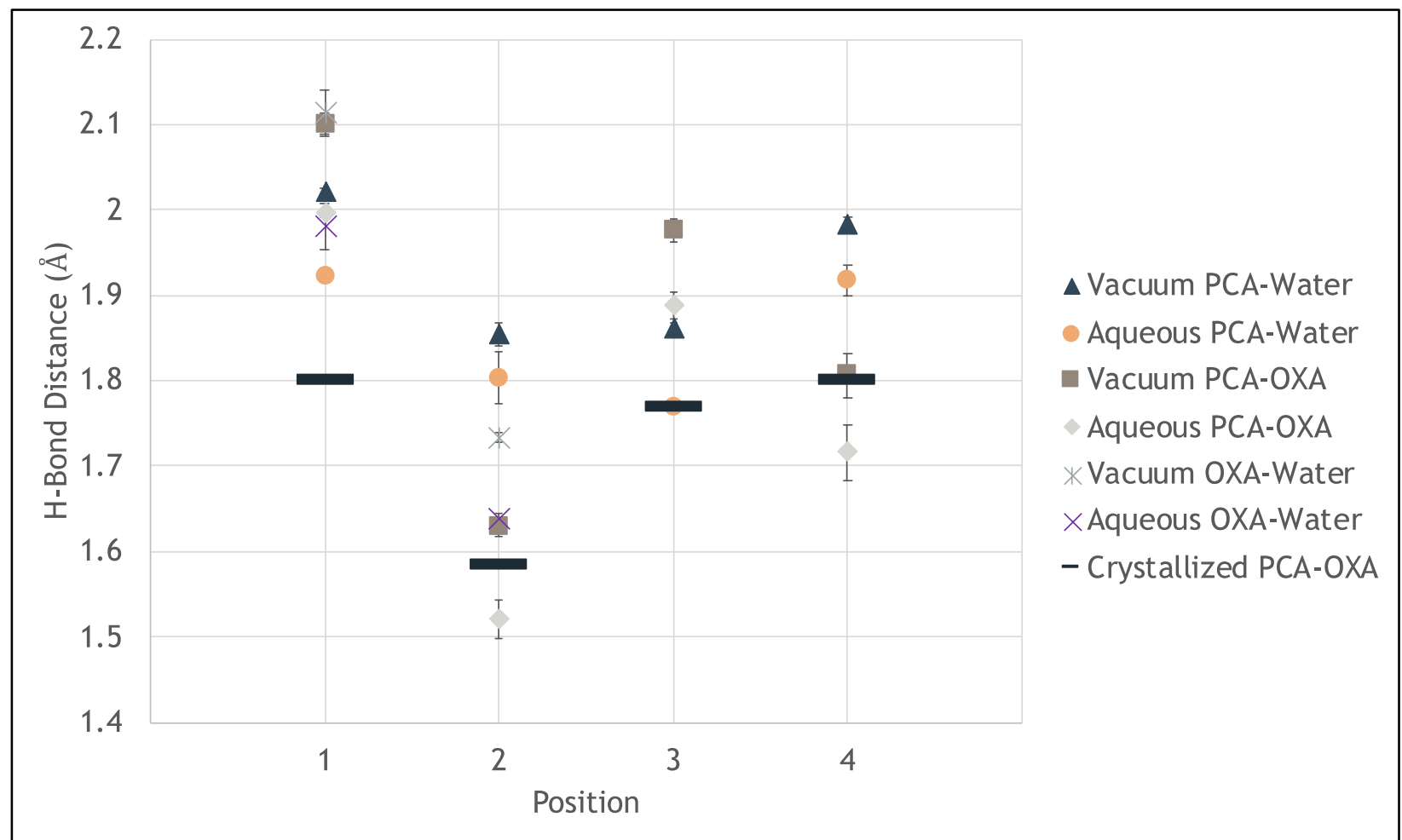

Figure 6. Averaged hydrogen bond lengths $(\AA)$ of the crystalized PCA-OXA and optimized PCA-Water and OXA-Water complexes in vacuum and an aqueous environment.

\subsection{BSSE Correction}

The BSSE was the greatest for PCA complexes at position $1(2.8-3.6 \mathrm{~kJ} / \mathrm{mol})$ as shown in Table 1 and Figure 7. This is a further indication that there is steric hindrance present between the aromatic ring and oxalic acid at this position. Overall BSSE values were smaller (1.7-3.6 kJ/mol) than nominal chemical accuracy $( \pm 4 \mathrm{~kJ} / \mathrm{mol})$. However, BSSE results do seem to provide some valuable insight into other possible intermolecular interactions present in the complexes and computational parameters. The standard deviation for BSSE ranged from $0.0 \mathrm{~kJ} / \mathrm{mol}$ to $0.1 \mathrm{~kJ} / \mathrm{mol}$ with the exception of $0.4 \mathrm{~kJ} / \mathrm{mol}$ for PCA-Water in an aqueous environment. The optimized geometries for this complex show variation in how the water is oriented with respect to PCA where 
Table 1. Complexation Energies (BSSE corrected) of PCA-OXA, PCA-Water, and OXA-Water Complexes $(\mathrm{kJ} / \mathrm{mol})$.

\begin{tabular}{|c|c|c|c|c|c|c|c|}
\hline & B3LYP-D3 & B3LYP-D3-BJ & M06-2X & M06-2X-D3 & $\omega \mathrm{B} 97-\mathrm{XD}$ & Average & Std \\
\hline & \multicolumn{7}{|c|}{ OXA-Water } \\
\hline Position 1 & -20.7 & -19.6 & -20.0 & -20.4 & -18.0 & -19.7 & 1.0 \\
\hline \multirow[t]{2}{*}{ Position 2} & -49.5 & -48.7 & -49.4 & -49.7 & -47.4 & -48.9 & 0.9 \\
\hline & \multicolumn{7}{|c|}{ Aqueous OXA-Water } \\
\hline Position 1 & -20.4 & -19.3 & -19.7 & -20.1 & -17.7 & -19.4 & 1.0 \\
\hline \multirow[t]{2}{*}{ Position 2} & -46.9 & -42.0 & -47.6 & -47.9 & -41.1 & -45.1 & 3.3 \\
\hline & \multicolumn{7}{|c|}{ PCA-Water } \\
\hline Position 1 & -25.9 & -24.8 & -25.7 & -26.4 & -24.9 & -25.5 & 0.7 \\
\hline Position 2 & -34.4 & -33.6 & -32.9 & -33.5 & -32.0 & -33.3 & 0.9 \\
\hline Position 3 & -29.8 & -29.1 & -28.9 & -29.3 & -28.7 & -29.2 & 0.4 \\
\hline \multirow[t]{2}{*}{ Position 4} & -21.2 & -20.1 & -19.9 & -20.4 & -18.5 & -20.0 & 1.0 \\
\hline & \multicolumn{7}{|c|}{ Aqueous PCA-Water } \\
\hline Position 1 & -24.7 & -23.6 & -24.6 & -25.4 & -23.7 & -24.4 & 0.7 \\
\hline Position 2 & -33.8 & -33.2 & -32.2 & -32.7 & -31.5 & -32.7 & 0.9 \\
\hline Position 3 & -29.7 & -29.0 & -28.6 & -29.0 & -28.6 & -29.0 & 0.4 \\
\hline \multirow[t]{2}{*}{ Position 4} & -20.1 & -18.3 & -19.0 & -17.2 & -17.9 & -18.5 & 1.1 \\
\hline & \multicolumn{7}{|c|}{ PCA-OXA } \\
\hline Position 1 & -21.8 & -20.4 & -17.7 & -19.8 & -18.7 & -19.7 & 1.5 \\
\hline Position 2 & -51.8 & -51.9 & -48.5 & -49.9 & -49.3 & -50.3 & 1.5 \\
\hline Position 3 & -28.1 & -27.5 & -24.9 & -26.4 & -25.4 & -26.5 & 1.4 \\
\hline \multirow[t]{2}{*}{ Position 4} & -33.7 & -33.1 & -30.9 & -32.0 & -31.7 & -32.3 & 1.1 \\
\hline & \multicolumn{7}{|c|}{ Aqueous PCA-OXA } \\
\hline Position 1 & -25.5 & -25.5 & -21.5 & -23.6 & -22.1 & -23.6 & 1.9 \\
\hline Position 2 & -69.9 & -69.7 & -58.4 & -59.8 & -64.8 & -64.5 & 5.4 \\
\hline Position 3 & -27.0 & -26.2 & -24.3 & -25.9 & -24.2 & -25.5 & 1.2 \\
\hline Position 4 & -39.8 & -39.7 & -43.2 & -44.4 & -37.3 & -40.9 & 2.9 \\
\hline
\end{tabular}

BSSE values are larger when the oxygen atom of water is oriented closer to the aromatic ring of PCA (Figure 7 and ESI). 


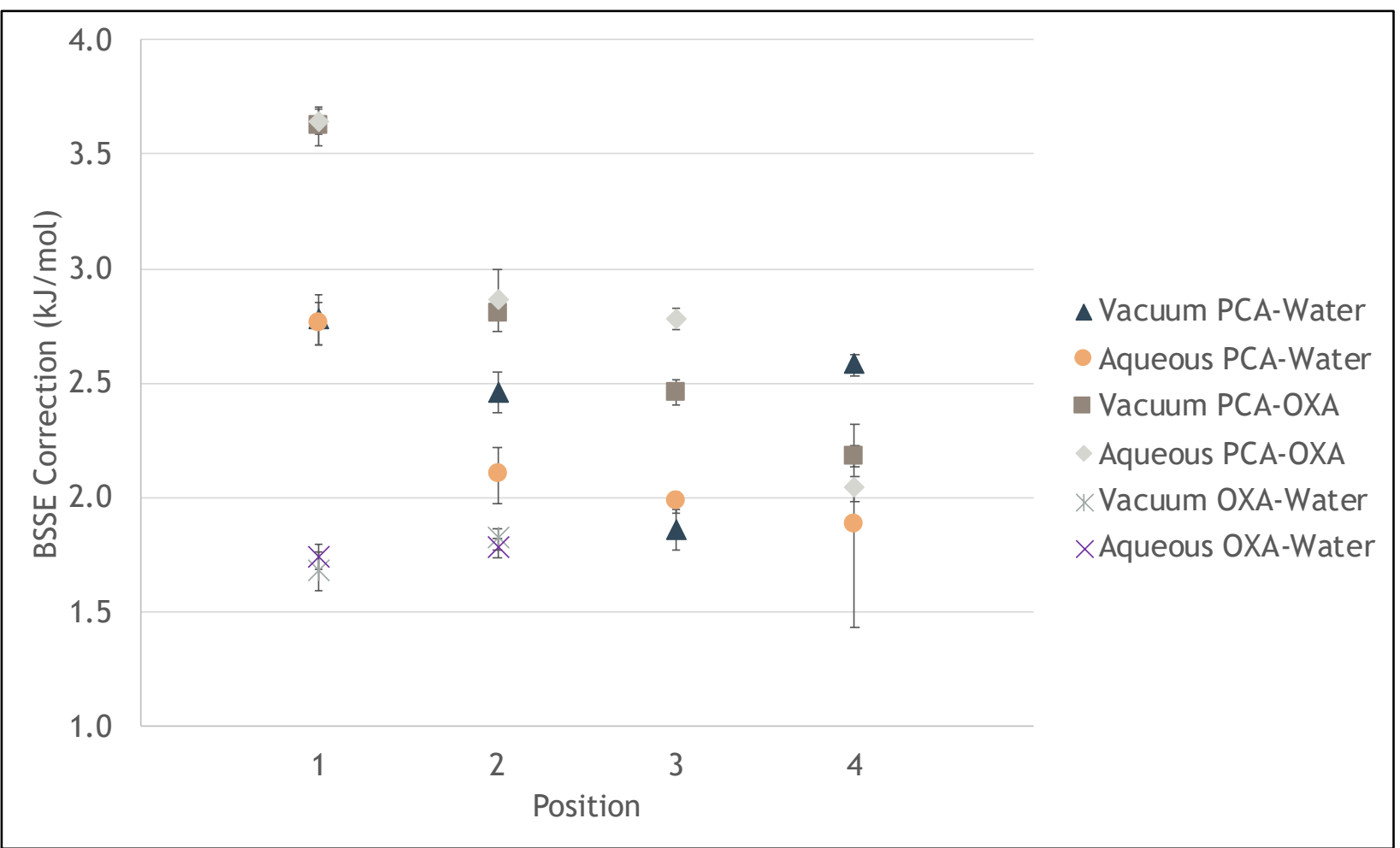

Figure 7. Averaged BSSE correction $(\mathrm{kJ} / \mathrm{mol})$ of the crystalized PCA-OXA and optimized PCA-Water and OXA-Water complexes in vacuum and an aqueous environment.

\subsection{Configuration Energy}

An evaluation of the configuration energies of the complexes can provide useful insights into the presence of energy barriers related to changes in molecular geometry as small molecular complexes build up into the actual crystalline structure. Changes in bond lengths and angles can alter torsional and steric strain within the molecules and any possible hindrance between molecules. The effect of implicit solvation had a clear impact on the configuration energies of PCA-OXA. After optimization of the complexes in vacuum, averaged configuration energies ranged from -67.1 to $-53.4 \mathrm{~kJ} / \mathrm{mol}$ as shown in Figure 8 . This difference shows that the energy of the relaxed structure is lower than that of the complexes in the crystal, indicating an energy barrier. Changes to bond lengths, angles, and molecular positions contribute to this change in energy. With complexation energies of -58.3 and $-67.1 \mathrm{~kJ} / \mathrm{mol}$, results indicate that positions 1 and 4 , 
respectively, are under the most geometric strain in the crystal configuration. These results agree with the geometrical analysis reported earlier. Results for position 4 are in line with earlier geometry observations because relaxed structures in the aqueous solvent do not form a secondary hydrogen bond. From the results obtained, the PCA-OXA complex is more stable in an aqueous environment, which means that there is a lower configuration energy barrier present for nucleation.

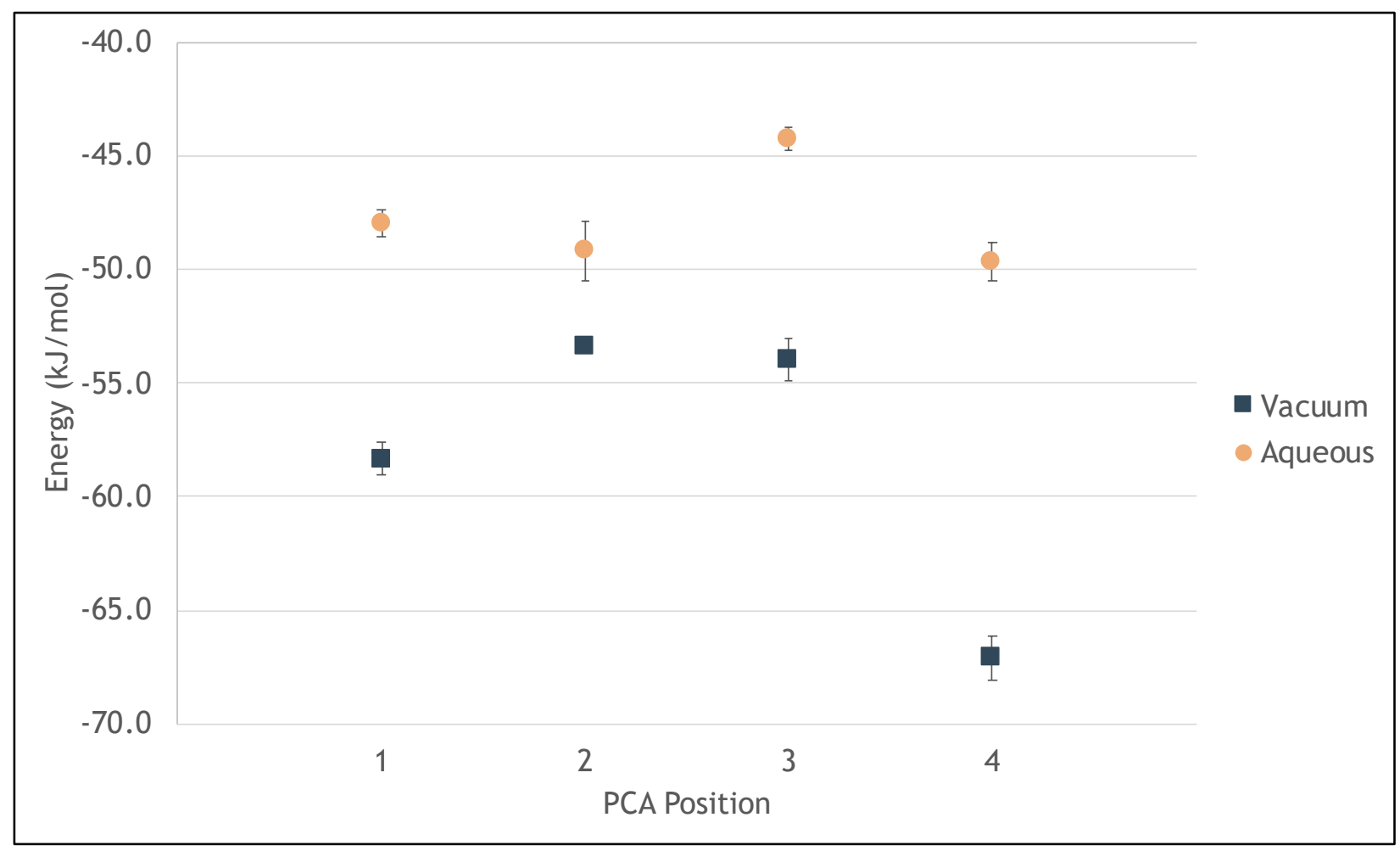

Figure 8. Averaged configuration energies $(\mathrm{kJ} / \mathrm{mol})$ of the PCA-OXA complexes in vacuum and an aqueous environment.

\subsection{Complexation Energy}

Complexation energies (BSSE corrected) were compared for the PCA-OXA, PCA-Water, and OXA-Water complexes in vacuum and with implicit solvation (Figure 9). In general, average complexation energies are strongest for the PCA-OXA complexes, with the lowest negative values observed at position $2(-50.3 \mathrm{~kJ} / \mathrm{mol}$ and $-64.5 \mathrm{~kJ} / \mathrm{mol}$ in vacuum and solvated environments, respectively) where oxalic acid acts as a hydrogen donor. Looking at all the complexes, it is evident 
that H-bonds with oxalic acid as the hydrogen donor results in stronger, more stable complexes. This observation is further supported by the complexation energies of OXA-Water at position 1, where oxalic acid acts as a hydrogen bond acceptor, vs position 2 , where oxalic acid is the hydrogen bond donor. On average, the complexation energies at position 2 were 29.2 and 25.5 $\mathrm{kJ} / \mathrm{mol}$ lower than at position 1 in vacuum and solvated environments, respectively. Based on these results, it is expected that the highly polar oxalic acid acting as a hydrogen donor is the main driving force for hydrogen bonding interactions with water and paracetamol.

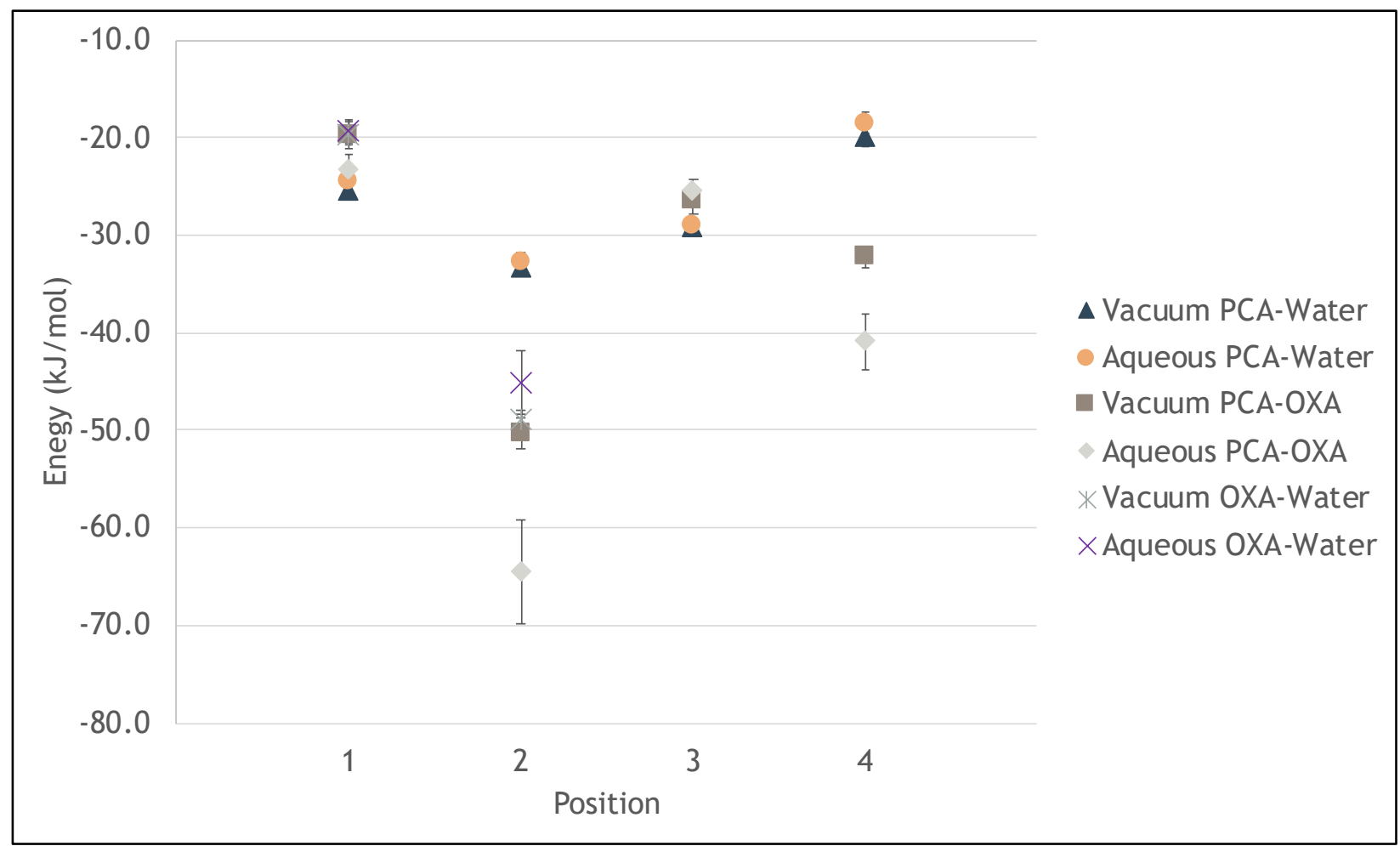

Figure 9. Averaged complexation energies $(\mathrm{kJ} / \mathrm{mol})$ of the PCA-OXA and optimized PCA-Water and OXA-Water complexes in vacuum and an aqueous environment.

Energy values for PCA-OXA were lower than those for OXA-Water, indicating that it is more energetically favorable for oxalic acid to form a hydrogen bond with PCA than with water. While PCA positions 1 and 3 show similar energetic trends, position 1 is not as energetically favorable. This could be due to the differences in polarity of the oxygens in the hydroxyl and amide functional groups. 


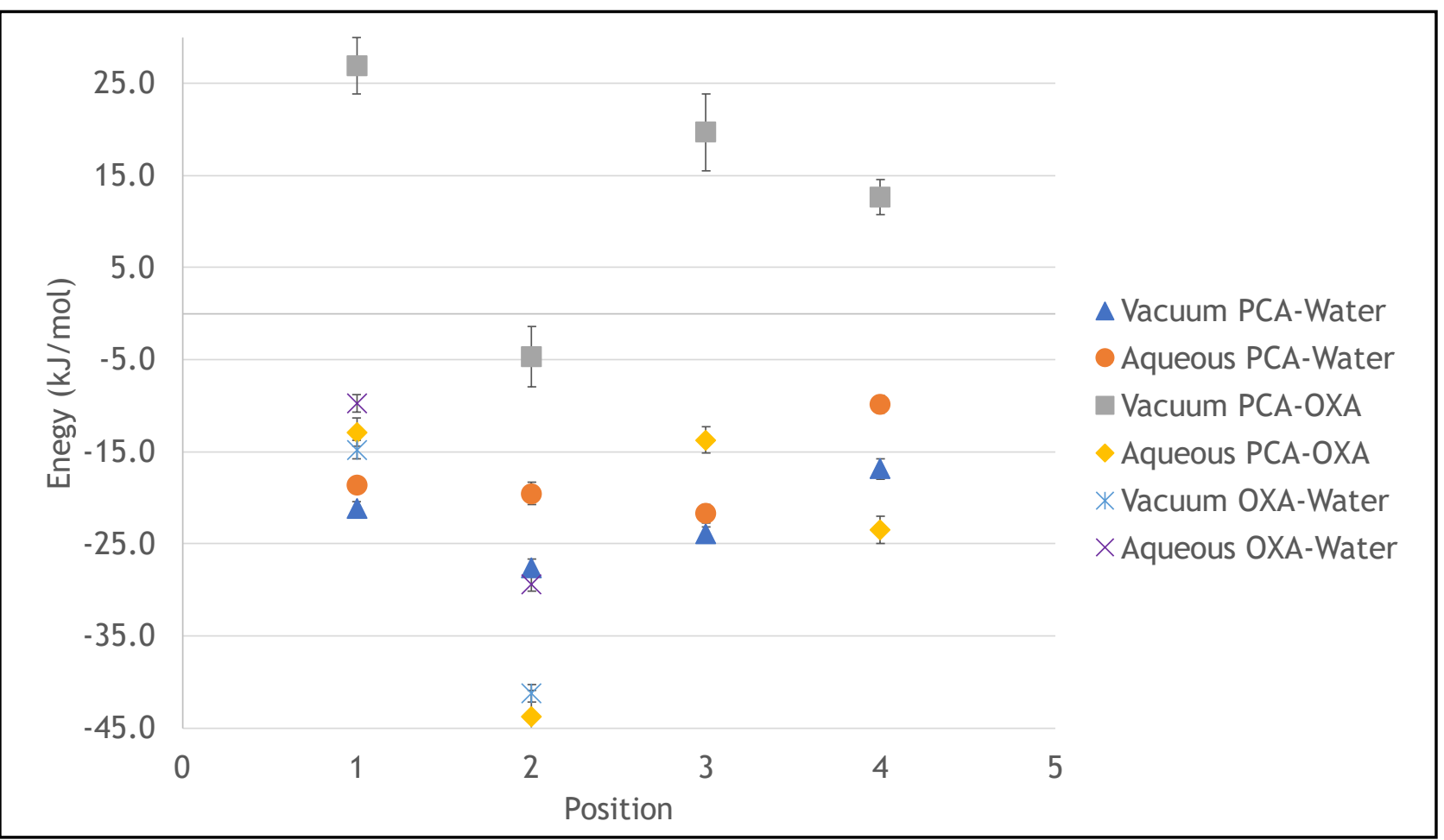

Figure 10. Averaged heats of complexation $(\mathrm{kJ} / \mathrm{mol})$ of the PCA-OXA and optimized PCA-Water and OXA-Water complexes in vacuum and an aqueous environment.

\subsection{Heats of Complexation}

The heats of complexation were compared for the PCA-OXA, PCA-Water, and OXAWater complexes in vacuum and with implicit solvation (Table 2, Figure 10). For the PCA-Water complexes, heats of complexation were lower in vacuum than in solvation, with the greatest differences noted at positions 2 and 4, with average values among the functionals of 8.1 and 7.0 $\mathrm{kJ} / \mathrm{mol}$, respectively. These results are in line with previously discussed results suggesting that in an aqueous environment, the PCA-Water complexes are less energetically favorable. For the PCAOXA complexes in vacuum, heats of complexation were positive at positions 1, 3, and 4 with position 1 having the highest heat of complexation of $+26.9 \mathrm{~kJ} / \mathrm{mol}$. At position 2 , the average heat of complexation was $-4.7 \mathrm{~kJ} / \mathrm{mol}$, indicating that only complexation at this site is energetically favorable in vacuum. While the overall trends in energy is consistent with all other findings 
Table 2. Heats of Complexation of PCA-OXA, PCA-Water, and OXA-Water Complexes (kJ/mol).

\begin{tabular}{|c|c|c|c|c|c|c|c|}
\hline & B3LYP-D3 & B3LYP-D3-BJ & M06-2X & M06-2X-D3 & $\omega B 97-X D$ & Average & Std \\
\hline & \multicolumn{7}{|c|}{ OXA-Water } \\
\hline Position 1 & -15.7 & -14.7 & -15.0 & -15.4 & -13.1 & -14.8 & 1.0 \\
\hline \multirow[t]{2}{*}{ Position 2} & -41.7 & -41.1 & -42.0 & -42.2 & -39.3 & -41.3 & 1.2 \\
\hline & \multicolumn{7}{|c|}{ Aqueous OXA-Water } \\
\hline Position 1 & -10.8 & -10.1 & -9.6 & -10.0 & -8.2 & -9.7 & 1.0 \\
\hline \multirow[t]{2}{*}{ Position 2} & -29.7 & -29.7 & -29.6 & -29.9 & -27.9 & -29.4 & 0.8 \\
\hline & \multicolumn{7}{|c|}{ PCA-Water } \\
\hline Position 1 & -21.6 & -20.5 & -21.3 & -22.1 & -20.4 & -21.2 & 0.7 \\
\hline Position 2 & -28.7 & -27.9 & -27.5 & -28.0 & -26.0 & -27.6 & 1.0 \\
\hline Position 3 & -24.1 & -23.4 & -23.3 & -23.7 & -25.2 & -23.9 & 0.8 \\
\hline \multirow[t]{2}{*}{ Position 4} & -16.5 & -15.6 & -17.8 & -18.3 & -16.2 & -16.9 & 1.1 \\
\hline & \multicolumn{7}{|c|}{ Aqueous PCA-Water } \\
\hline Position 1 & -19.2 & -18.1 & -18.4 & -19.2 & -18.1 & -18.6 & 0.6 \\
\hline Position 2 & -20.1 & -19.7 & -20.0 & -20.5 & -17.4 & -19.5 & 1.2 \\
\hline Position 3 & -21.0 & -20.4 & -22.4 & -22.9 & -22.0 & -21.7 & 1.0 \\
\hline \multirow[t]{2}{*}{ Position 4} & -11.1 & -10.7 & -9.0 & -9.5 & -9.2 & -9.9 & 0.9 \\
\hline & \multicolumn{7}{|c|}{ PCA-OXA } \\
\hline Position 1 & +22.3 & +25.3 & +30.1 & +28.0 & +28.6 & +26.9 & 3.1 \\
\hline Position 2 & -7.8 & -8.7 & -1.7 & -3.2 & -2.0 & -4.7 & 3.3 \\
\hline Position 3 & +14.1 & +16.3 & +23.5 & +22.0 & +22.4 & +19.7 & 4.2 \\
\hline \multirow[t]{2}{*}{ Position 4} & +10.8 & +10.5 & +14.8 & +13.7 & +13.6 & +12.7 & 1.9 \\
\hline & \multicolumn{7}{|c|}{ Aqueous PCA-OXA } \\
\hline Position 1 & -15.0 & -13.5 & -11.1 & -13.1 & -11.8 & -12.9 & 1.5 \\
\hline Position 2 & -46.8 & -46.9 & -40.7 & -42.1 & -42.4 & -43.8 & 2.9 \\
\hline Position 3 & -15.6 & -14.8 & -12.1 & -13.6 & -12.6 & -13.7 & 1.5 \\
\hline Position 4 & -25.0 & -24.9 & -22.2 & -23.4 & -21.9 & -23.5 & 1.4 \\
\hline
\end{tabular}


(energies at positions 1 and 3 are higher than positions 2 and 4), it is difficult to conclude if enthalpies of formation are actually positive, or if the chosen methods and basis set aren't able to accurately describe this particular system. Heats of complexation for PCA-OXA in an aqueous environment were all negative and showed the same trends in energy as observed throughout the overall sets of results where positions 1 and 3 were comparably similar -12.9 and $-13.7 \mathrm{~kJ} / \mathrm{mol}$ ), and positions 2 and 4 were more energetically favorable, with position 2 having the most stable energy $(-23.5 \mathrm{~kJ} / \mathrm{mol}$ at position 4 and $-43.8 \mathrm{~kJ} / \mathrm{mol}$ at position 2$)$. When comparing OXA-Water in an aqueous environment vs vacuum, enthalpies of complexation were lowest at position 2 in both vacuum and aqueous $(-41.3$ and $-29.4 \mathrm{~kJ} / \mathrm{mol})$ and were lower in vacuum by $5.1 \mathrm{~kJ} / \mathrm{mol}$ at position 1 and $11.9 \mathrm{~kJ} / \mathrm{mol}$ at position 2 which again is consistent with trends found for complexation energies. Overall, results for heats of complexation supported the trends found for complexation and configuration energies showing that position 2 is the most energetically favorable site for PCA-OXA complexation.

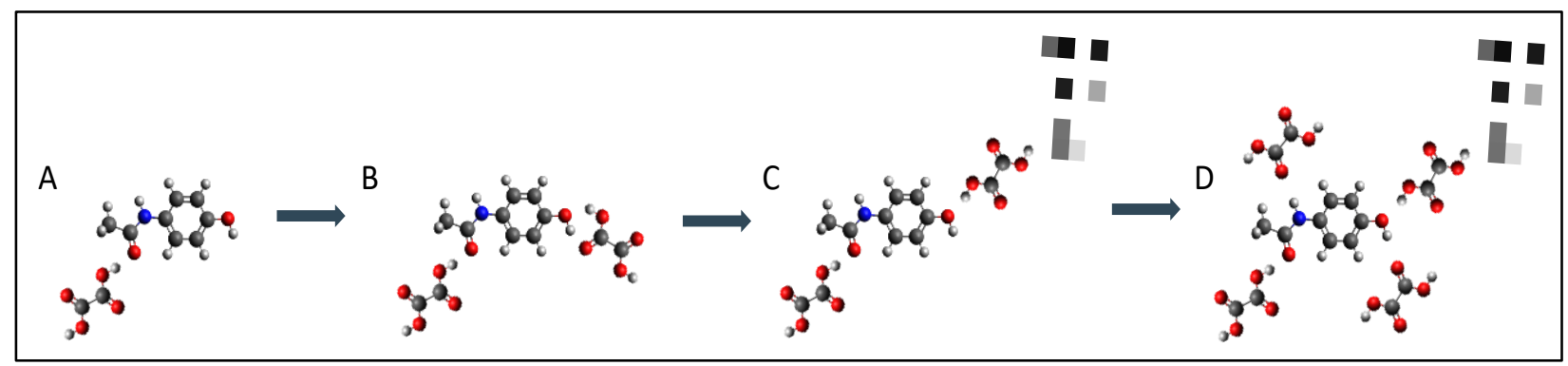

Figure 11. Proposed nucleation path for the PCA-OXA complex. Nucleation begins with (A) hydrogen bonding of oxalic acid as the hydrogen donor to the amide followed by (B) the hydroxyl functional groups of PCA with (C) additional hydrogen bonding of oxalic acid being dominated by the amide of other PCA molecules. Weak hydrogen bonding of oxalic acid as the hydrogen bond acceptor with the (D) amide and hydroxyl groups completes the crystal structure. 


\section{Conclusions}

Optimization calculations for PCA-OXA at position 3 have proved to be challenging due in part to the shallow potential energy well and to the steric strain present from the aromatic ring on paracetamol in the crystallized geometry. Results show that complexation of PCA-OXA is driven by hydrogen bonding at PCA positions 2 and 4, with position 2 showing the most stable intermolecular interactions. From these results, it is proposed that nucleation is driven by hydrogen bonding at position 2 of PCA. Figure 11 shows the proposed nucleation path for the PCA-OXA crystal. The hydrogen donor site of oxalic acid provides a more energetically favorable complex than water and the stability gained from the formation of the hydrogen bonds outweighs the conformation strain of the PCA-OXA complex during crystallization at positions 1 and 3.

In an aqueous environment, the complexation energies of PCA-Water were slightly weaker $(0.2-1.5 \mathrm{~kJ} / \mathrm{mol})$ than in vacuum. This suggests that water has a higher affinity for itself than it does for PCA. The same trend is seen with heats of complexation. It is expected that in an aqueous environment, there would be little to no energetic competition for PCA to form hydrogen bonds with other molecules. This is particularly important in regards to the pharmacodynamics of PCA's ability to interact with the receptor binding site in vivo. The same trend was noted with the OXAWater complex, which again suggests little to no energetic competition of oxalic acid to form hydrogen bonds with other molecules in an aqueous environment such as the water-filled pore spaces in soil.

For the PCA-OXA complex, complexation energies were lower by 3.3, 14.2, and 8.6 $\mathrm{kJ} / \mathrm{mol}$ at positions 1,2 , and 4 respectively in an aqueous environment. This suggests that, overall, the PCA-OXA complex is more stable in an aqueous environment than in a vacuum. This can be related to the increased polarity of oxalic acid and PCA that is induced with the presence of water. 
The most notable difference is seen at positions 2 and 4 with differences in complexation energies of -15.0 and $-9.1 \mathrm{~kJ} / \mathrm{mol}$ respectively and heats of complexation of -39.1 and $-36.2 \mathrm{~kJ} / \mathrm{mol}$. At these positions, oxalic acid acts as the hydrogen donor. With position 3, complexation energies were slightly higher in an aqueous environment. Here PCA acts as the hydrogen donor. These results suggest that in an aqueous environment, complexes are strengthened when the more polar species participates in hydrogen bonding as a hydrogen donor. These results are specifically insightful when trying to determine the effects of a solvated environment and hydrogen bonding, with particular attention to the relative polarities and the role of each species in the complex.

Overall, the five different functionals employed provided similar results with the most variation seen for PCA complexes in positions 2 and 4 that were affected both by the functional used (std 5.4 and 2.9 respectively in an aqueous environment) and the environment (-14.2 and -8.6 $\mathrm{kJ} / \mathrm{mol}$ differences for positions 2 and 4 in vacuum vs aqueous). Heats of complexation for PCAOXA in vacuum were very high compared to all other heats of complexation with positive values at positions 1,3 , and 4 . It is possible that the functionals and/or basis set used did not provide accurate results for this specific system. Further comparison with empirical data or investigation of the potential energy surface could provide insight to these results. Variations in energies were consistent with the degree of rotation of either water or oxalic acid with respect to PCA, with larger rotations resulting in more dramatic differences in energy among the functionals.

While an in-depth study into the hydrogen bonding of the PCA-OXA complex yields tremendous insight into the behavior behind complexation, there are several variables that are also expected to play a role in the complexation and eventual nucleation of the PCA-OXA crystal. Future work will include investigation of larger PCA-OXA molecular complexes including $\pi-\pi$ 
interlayer interactions, analysis of the HOMO-LUMO gaps to explore chemical reactivity, infrared and nuclear magnetic resonance (NMR) spectral shifts, and 1D-potential energy surfaces.

\section{Acknowledgments}

The authors acknowledge the Department of Chemistry at Michigan Technological University for lab space. AS acknowledges the Department of Chemistry at Michigan Technological University for support. The High-Performance Computing Shared Facility Superior at Michigan Technological University was used in obtaining results presented in this publication.

\section{Available Supporting Information:}

Table S-1: Effect of optimization and frequency calculation parameter changes on results for PCAWater.

Table S-2: Molecular bond distances and angles for oxalic acid.

Table S-3: Molecular bond distances and angles for paracetamol.

Figure S-1: Geometry changes of PCA-Water in vacuum and aqueous environments.

Figure S-2: Geometry comparison across functionals for PCA-OXA at position 4. 


\section{References}

1. Wouters, J.; Quéré, L., Pharmaceutical salts and co-crystals. Royal Society of Chemistry: 2011.

2. Monteiro, S. C.; Boxall, A. B., Factors affecting the degradation of pharmaceuticals in agricultural soils. Environmental Toxicology and Chemistry 2009, 28 (12), 2546-2554.

3. CSD web interface - intuitive, cross-platform, web-based access to CSD data. Cambridge Crystallographic Data Centre. CCDS: 12 Union Road, Cambridge, UK, 2017.

4. M. J. Frisch, G. W. T., H. B. Schlegel, G. E. Scuseria, M. A. Robb, J. R. Cheeseman, G. Scalmani, V. Barone, G. A. Petersson, H. Nakatsuji, X. Li, M. Caricato, A. V. Marenich, J.

Bloino, B. G. Janesko, R. Gomperts, B. Mennucci, H. P. Hratchian, J. V. Ortiz, A. F. Izmaylov, J. L. Sonnenberg, D. Williams-Young, F. Ding, F. Lipparini, F. Egidi, J. Goings, B. Peng, A. Petrone, T. Henderson, D. Ranasinghe, V. G. Zakrzewski, J. Gao, N. Rega, G. Zheng, W. Liang, M. Hada, M. Ehara, K. Toyota, R. Fukuda, J. Hasegawa, M. Ishida, T. Nakajima, Y. Honda, O. Kitao, H. Nakai, T. Vreven, K. Throssell, J. A. Montgomery, Jr., J. E. Peralta, F. Ogliaro, M. J. Bearpark, J. J. Heyd, E. N. Brothers, K. N. Kudin, V. N. Staroverov, T. A. Keith, R. Kobayashi, J. Normand, K. Raghavachari, A. P. Rendell, J. C. Burant, S. S. Iyengar, J. Tomasi, M. Cossi, J. M. Millam, M. Klene, C. Adamo, R. Cammi, J. W. Ochterski, R. L. Martin, K. Morokuma, O. Farkas, J. B. Foresman, and D. J. Fox Gaussian 16, Revision A.03, Gaussian, Inc.: Wallingford CT, 2016.

5. Mintmire, J.; Labanowski, J.; Andzelm, J., Density Functional Methods in Chemistry. 1991.

6. Becke, A. D., Density-functional thermochemistry. I. The effect of the exchange-only gradient correction. The Journal of chemical physics 1992, 96 (3), 2155-2160. 
7. Becke, A. D., Density-functional thermochemistry. II. The effect of the Perdew-Wang generalized-gradient correlation correction. The Journal of chemical physics 1992, 97 (12), 9173-9177.

8. Becke, A., Density2Functional Thermochemistry III. The Role of Extract Exchange. $J$ Chem Phys 1993, 98 (5), 648-5.

9. Zhao, Y.; Truhlar, D. G., The M06 suite of density functionals for main group thermochemistry, thermochemical kinetics, noncovalent interactions, excited states, and transition elements: two new functionals and systematic testing of four M06-class functionals and 12 other functionals. Theoretical Chemistry Accounts 2008, 120 (1), 215-241.

10. Chai, J.-D.; Head-Gordon, M., Long-range corrected hybrid density functionals with damped atom-atom dispersion corrections. Physical Chemistry Chemical Physics 2008, 10 (44), $6615-6620$.

11. Grimme, S.; Ehrlich, S.; Goerigk, L., Effect of the damping function in dispersion corrected density functional theory. Journal of computational chemistry 2011, 32 (7), 14561465.

12. Becke, A. D.; Johnson, E. R., Exchange-hole dipole moment and the dispersion interaction revisited. The Journal of chemical physics 2007, 127 (15), 154108.

13. Dunning Jr, T. H., Gaussian basis sets for use in correlated molecular calculations. I. The atoms boron through neon and hydrogen. The Journal of chemical physics 1989, 90 (2), 10071023.

14. Barone, V.; Cossi, M., Quantum calculation of molecular energies and energy gradients in solution by a conductor solvent model. The Journal of Physical Chemistry A 1998, 102 (11), 1995-2001. 
15. Kállay, M.; Surján, P. R., Higher excitations in coupled-cluster theory. The Journal of Chemical Physics 2001, 115 (7), 2945-2954.

16. Simon i Rabasseda, S.; Duran i Portas, M.; Dannenberg, J., How does basis set superposition error change the potential surfaces for hydrogen-bonded dimers? (C) Journal of Chemical Physics, 1996, vol. 105, núm. 24, p. 11024-11031 1996.

17. Karki, S.; Friščić, T.; Fábián, L.; Laity, P. R.; Day, G. M.; Jones, W., Improving mechanical properties of crystalline solids by cocrystal formation: new compressible forms of paracetamol. Advanced materials 2009, 21 (38-39), 3905-3909.

18. P. Ugliengo, D. V., G. Chiari Z. Kristallogr MOLDRAW: Molecular Graphics on a Personal Computer, 9; 1993.

19. Hanwell, M. D.; Curtis, D. E.; Lonie, D. C.; Vandermeersch, T.; Zurek, E.; Hutchison, G. R., Avogadro: an advanced semantic chemical editor, visualization, and analysis platform. Journal of cheminformatics 2012, 4 (1), 17. 